\title{
REMARKS ON RECENT FIXED POINT THEOREMS FOR COMPATIBLE MAPS
}

\author{
S.L. SINGH \\ Department of Mathematics \\ Gurukula Kangri University \\ Hardwar 249404, INDIA \\ V. CHADHA \\ Department of Mathematics \\ University of Wisconsin \\ Eau Claire, WI 54702, USA \\ S.N. MISHRA \\ Department of Mathematics \\ Private Bag XI Unitra, Umtata \\ Republic of Transkei, SOUTHERN AFRICA
}

(Received November 22, 1994 and in revised form August 3, 1995)

\begin{abstract}
Following Gerald Jungck's formulation of compatibility of maps, several interesting fixed point theorems for such maps have been obtained, wherein at least one of the maps is continuous The main purpose of this note is to avoid the continuity condition from some of the main results of Jungck et al
\end{abstract}

KEY WORDS AND PHRASES: Common fixed points, coincidence points, compatible maps, biased maps, $(\epsilon, \delta)$-contractions

1991 AMS SUBJECT CLASSIFICATION CODES: 54H25

\section{INTRODUCTION}

Self-maps $f$ and $g$ of a metric space $(X, d)$ are compatible iff $\lim _{n} d\left(f g x_{n}, g f x_{n}\right)=0$ whenever $\left\{x_{n}\right\}$ is a sequence in $X$ such that $f x_{n}, g x_{n} \rightarrow t$ for some $t$ in $X$ (cf [1]-[2], see also [3]-[10]) Compatible maps are natural generalizations of commuting and weakly commuting maps [11] (see for instance [1]-[10]) Very recently a less restrictive concept called "biased maps" has been studied by Jungck and Pathak [6] Let $A$ and $S$ be self-maps of a metric space $(X, d)$ The pair $\{A, S\}$ is $S$-biased iff whenever $\left\{x_{n}\right\}$ is a sequence in $X$ and $A x_{n}, S x_{n} \rightarrow t \in X$, then

$$
\alpha d\left(S A x_{n}, S x_{n}\right) \leq \alpha d\left(A S x_{n}, A x_{n}\right) \text { if } \alpha=\text { liminf and if } \alpha=\text { limsup. }
$$

They have shown that if the pair $\{A, S\}$ is compatible, then it is both $S$ and $A$-biased (see [6], Remark 11)

Recently several fixed point theorems for compatible maps have been obtained ([1]-[10] and references of [7]), and a few of them have been extended to biased maps [6] However, most of the results, especially those for compatible maps, require continuity of at least one of the maps ([1]-[5], [7][10]) In this note, we present fixed point theorems for four maps, wherein the continuity condition is not needed Our results are variants of some interesting results, mainly, of Jungck Indeed, motivation to waive the continuity condition referred to above comes from [12]-[14] and [6]

\section{2. $(\epsilon, \delta)$-CONTRACTION FOR FOUR MAPS}

Meir-Keeler type contractions for four maps on a metric space introduced in [1] have been further studied in [4]-[6], [10] and elsewhere These motivate the following, where $(X, d)$ is a metric space 
DEFINITION 1. A pair of maps $A$ and $B$ from $X$ to itself are $(\epsilon, \delta)$-contractions relative to maps $S, T: X \rightarrow X$ iff $A(X) \subseteq T(X), B(X) \subseteq S(X)$, and there exists a function $\delta:(0, \infty) \rightarrow(0, \infty)$ such that $\delta(\epsilon)>\epsilon$ for all $\epsilon$, and for $x, y$ in $X$

(i) $\epsilon \leq m(x, y)<\delta(\epsilon)$ implies that $d(A x, B y)<\epsilon$, and

(ii) $A x=B y$ whenever $S x=T y$,

wherein

$$
m(x, y)=\max \left\{d(S x, T y), k d(S x, A x), k d(T y, B y), \frac{1}{2}[d(S x, B y)+d(A x, T y)]\right\} .
$$

where $k$ is constant such that $0 \leq k<1$

Of interest to us is the following result which is a variant of the main result of [6] and improves several earlier results from [1], [4], [12] and [15] In all that follows $C(A, S)$ stands for the collection of coincidence points of $A$ and $S$, i e, $C(A, S)=\{u: A u=S u\}$ We shall use $N$ to denote the positive integers

THEOREM 1. Let $(X, d)$ be a metric space, $S, T: X \rightarrow X$, and let $A, B$ be $(\epsilon, \delta)-(S, T)$ contractions (cf Definition 1 above) If one of $A(X), B(X), S(X)$ or $T(X)$ is a complete subspace of $X$, then

$$
\begin{aligned}
& A \text { and } S \text { have a coincidence, } \\
& B \text { and } T \text { have a coincidence }
\end{aligned}
$$

Further, if $A S u=S A u, u \in C(A, S)$ and $B T v=T B v, v \in C(B, T)$, then

$$
A, B, S \text { and } T \text { have a unique common fixed point }
$$

PROOF. Pick $x_{0} \in X$ and, akin to [4] and [6], construct two sequences $\left\{x_{n}\right\} \subset X$ and $\left\{y_{n}\right\} \subset X$ in the following manner For $n$ in $N$,

$$
y_{2 n-1}=T x_{2 n-1}=A x_{2 n-2} \text { and } y_{2 n}=S x_{2 n}=B x_{2 n-1}
$$

Then, as in [10] (see also [5] and [6]), $\left\{y_{n}\right\}$ is a Cauchy sequence

Now suppose $S(X)$ is complete Then the subsequence $\left\{y_{2 n}\right\}$ being contained in $S(X)$ has a limit in $S(X) \quad$ Call it $u$. Let $v \in S^{-1} u$ Then $S v=u$ Note that the subsequence $\left\{y_{2 n-1}\right\}$ also converges to $u$ Since $A, B$ are $(\epsilon, \delta)-(S, T)$-contractions,

$$
\begin{aligned}
& d(A x, B y) \leq m(x, y) \text { for any } x, y \text { in } X, \text { and } \\
& A x \neq B y \text { implies that } d(A x, B y)<m(x, y)
\end{aligned}
$$

(see [6], Remark 2 1). Putting $x=v$ and $y=x_{2 n-1}$ in (14),

$$
\begin{aligned}
d\left(A v, y_{2 n}\right)=d\left(A v, B x_{2 n-1}\right) \leq \max \left\{d\left(S v, T x_{2 n-1}\right), k d(S v, A v), k d\left(T x_{2 n-1}, B x_{2 n-1}\right),\right. \\
\left.\frac{1}{2}\left[d\left(S v, T x_{2 n-1}\right)+d\left(A v, T x_{2 n-1}\right)\right]\right\}
\end{aligned}
$$

and taking the limit as $n \rightarrow \infty, d(A v, u) \leq k d(A v, u)$ So $A v=u$ This proves (1 1 ).

Since $A(X) \subseteq T(X)$, there is an element $w$ in $X$ such that $A v=T w$, i e, $T w=u$ Therefore, as previously (or putting $x=x_{2 n-2}$ and $y=w$ in (14) and passing to the limit), $B w=u$ This proves (1 2 )

If we suppose that $T(X)$ is complete then analogous argument establishes (ll 1 ) and (1 2) If $B(X)$ (resp $A(X)$ ) is complete, then $u \in B(X) \subset S(X)$ (resp $u \in A(X) \subset T(X)$ ), and the above argument establishes (1 11$)$ and (1 2$)$.

To prove (1.3), note that $v \in C(A, S), w \in C(B, T)$ and

(a) $A v=S v=u=B w=T w$,

(b) $A u=A S v=S A v=S u$,

(c) $B u=B T w=T B w=T u$

If $u \neq B u$ then from (14), 


$$
\begin{aligned}
d(u, B u) & =d(A v, B u)<\max \left\{d(S v, T u), \frac{1}{2}[d(S v, B u)+d(A v, T u)]\right\} \\
& =d(u, B u),
\end{aligned}
$$

a contradiction So $u=B u$ Similarly, $u=A u$

Using (b)-(c) one finds that $u$ is a common fixed point of $A, B, S$ and $T$ The uniqueness of the common fixed point follows easily

REMARK 1. If $S=T$ in Theorem 1 , then conclusions (1 1 )-(ll 12 ) are slightly improved to " $A, B$ and $T$ have a coincidence, $\mathrm{i} \mathrm{e}$, there is a $z$ in $Y$ such that $A z=B z=T z$ " Then the statement concerning conclusion (1 3) may be improved to "Further, if $T(=S)$ commutes with each of $A$ and $B$ (only at) $z$, then $A, B$ and $T$ have a unique common fixed point "

\section{COMMON FIXED POINT THEOREMS FOR JUNGCK TYPE CONTRACTIONS}

Following the main theme behind the above proof, several common fixed point theorems from [2], [6]-[8] and [13]-[14] for maps satisfying Jungck type contractions may be improved In this section we show that the same behavior is exhibited for certain general classes of maps

Throughout this section, suppose that the function $\phi:[0, \infty)^{5} \rightarrow[0, \infty)$ satisfies the following conditions

(I) $\phi$ is nondecreasing and upper semicontinuous in each coordinate variable,

(II) for each $t>0, \max \{\phi(0,0, t, t, t), \phi(t, t, t, 2 t, 0), \phi(t, t, t, 0,2 t)\}<t$

The following result improves the main result of [8] by removing the assumption of continuity, and replacing the completeness of the space with a set of weaker conditions

THEOREM 2. Let $(X, d)$ be a metric space, and $A, B, S, T: X \rightarrow X$ such that $A(X) \subseteq T(X)$, $B(X) \subseteq S(X)$ and the following holds

$$
d(A x, B y) \leq \phi(d(A x, S x), d(B y, T y), d(S x, T y), d(A x, T y), d(B y, S x))
$$

for all $x, y$ in $X$, wherein $\phi$ satisfies (I) and (II) If one of $A(X), B(X), S(X)$ or $T(X)$ is a complete subspace of $X$, then

$$
\begin{aligned}
& A \text { and } S \text { have a coincidence, } \\
& B \text { and } T \text { have a coincidence }
\end{aligned}
$$

Further, if $A S u=S A u, u \in C(A, S)$ and $B T v=T B v, v \in C(B, T)$, then

$$
A, B, S \text { and } T \text { have a unique common fixed point }
$$

PROOF. For an $x_{0} \in X$ and $n \in N$, construct sequences $\left\{x_{n}\right\}$ and $\left\{y_{n}\right\}$ as in the proof of Theorem 1 Then, as in [8, Theorem 22], it can be shown that $\left\{y_{n}\right\}$ is a Cauchy sequence The remainder of the proof is not difficult, and is similar to that of Theorem 1. However, we shall sketch the proof to establish (2 1) when $S(X)$ is assumed to be complete. Since $\left\{y_{n}\right\}$ is Cauchy, the subsequence $\left\{y_{2 n}\right\}$ has a limit in $S(X)$. Call it $u$ Then $u=S v$ for some $v$ in $X$ Remembering that $A x_{2 n}, S x_{2 n}, B x_{2 n-1}, T x_{2 n-1} \rightarrow u$, putting $x=v$ and $y=x_{2 n-1}$ in (20) and passing to the limit, one obtains

$$
d(A v, u) \leq \phi(d(A v, u), 0,0, d(A v, u), 0)<d(A v, u),
$$

a contradiction unless $A v=u(=S v)$ This establishes (2 1$)$

From Theorem 2, defining $\phi:[0, \infty)^{5} \rightarrow[0, \infty)$ by $\phi\left(t_{1}, t_{2}, t_{3}, t_{4}, t_{5}\right)=q \max \left\{t_{1}, t_{2}, t_{3},\left(t_{4}+t_{5}\right) / 2\right\}$ for $q \in(0,1)$ and all $t_{2} \in[0, \infty), i=1, \ldots, 5$, we obtain the following improvement of Jungck [2] (see also [3], Theorem 3.1)

COROLLARY 1. Let $(X, d)$ be a metric space, and $A, B, S, T: X \rightarrow X$ such that $A(X) \subseteq T(X), B(X) \subseteq S(X)$ and the following holds

$$
d(A x, B y) \leq q \max \left\{\left(d(A x, S x), d(B y, T y), d(S x, T y), \frac{1}{2}[d(A x, T y)+d(B y, S x)]\right)\right\}
$$


for all $x, y \in X$, where $q \in(0,1)$ If one of $A(X), B(X), S(X)$ or $T(X)$ is a complete subspace of $X$, then the conclusions (21) and (22) are true Further, pairs $\{A, S\}$ and $\{B, T\}$ commute at their concidence points, then $(23)$ is also true

REMARK 2. If $S=T$ in Theorems 2-3, then their conclusions (2 1 1)-(2 3 ) can easily be improved to "There exists a point $z$ in $Y$ such that $A z=B z=T z \quad$ Further, if $T$ commutes with each of $A$ and $B$ at (only) $z$, then $A, B$ and $T$ have a unique common fixed point "

Finally we present an improvement of a common fixed point theorem for three maps of JungckRhoades [7, Theorem 2 3]

THEOREM 3. Let $f, g$ and $h$ be self-maps of a metric space $X$ such that $f(X) \cup g(X) \subseteq h(X)$ Suppose that

$$
[d(f x, g y)]^{2} \leq \psi(d(h x, f x) d(h y, g y), d(h x, g y) d(h y, f x), d(h x, f x) d(h x, g y), d(h y, f x) d(h y, g y))
$$

for any $x, y \in X$, where $\Psi:[0, \infty)^{4} \rightarrow[0, \infty)$ is upper semi-continuous and nondecreasing in each coordinate variable and satisfies $\Psi\left(t, t, a_{1} t, a_{2} t\right)<t$ for any $t>0$, where $a_{1}, a_{2} \in\{0,1,2\}$ with $a_{1}+a_{2}=2$ If one of $f(X), g(X)$ or $h(X)$ is a complete subspace of $X$ then $f, g$ and $h$ have a coincidence, i e, there exists a $z$ in $X$ such that $f z=g z=h z$ Further, if $h$ commutes with each of $f$ and $g$ (just at one point) $z$, then $f, g$ and $h$ have a unique common fixed point

PROOF. It may be completed following the proof of Theorem 1 (above), [7] and [12]-[14]

ACKNOWLEDGMENT. This work was completed while the first author (S L Singh) was visiting the University of Wisconsin, Eau Claire during the summer of 1994 He thanks Professor David R Lund, Professor Shyam S Chadha and the University for their hospitality and support The authors thank the referee for his appreciation and valuable suggestions to improve upon the original version of this paper

\section{REFERENCES}

[1] JUNGCK, G, Compatible mappings and common fixed points, Internat. J. Math. Math. Scl. 9 (1986), 771-779

[3] JUNGCK, G, Compatible mappings and common fixed points (2), Internat. J. Math. Math. Scl. 9 (1986), 285-288

[4] JUNGCK, G, Common fixed points for commuting and compatible maps on compacta, Proc. Amer. Math. Soc. 103 (1988), 977-983

[4] JUNGCK, G, Compatible mappings and common fixed points "revisited," Internat. J. Math. Math. Scl. 17 (1994), 37-40

[5] JUNGCK, G, MOON, K B , PARK, S and RHOADES, B E , On generalizations of the MeirKeeler type contraction maps correction, J. Math. Anal. Appl. 180 (1993), 221-222

[6] JUNGCK, G and PATHAK, H K, Fixed points via "biased maps," Proc. Amer. Math. Soc. (to appear)

[7] JUNGCK, G and RHOADES, B E, Some fixed point theorems for compatible maps, Internat. J. Math. Math. Sci. 16 (1993), 417-428

[8] KANG, S.M., CHO, Y J. and JUNGCK, G, Common fixed points of compatible mappings, Internat. J. Math. Math. Scl. 13 (1990), 61-66

[9] KANG, S M and RHOADES, B E , Fixed points for four mappings, Math. Japon. 37 (1992), 1053 1059

[10] RHOADES, B.E, PARK, S and MOON, K B , On generalizations of Meir-Keeler type contraction maps, J. Math. Anal. Appl. 146 (1990), 482-494

[11] SESSA, S, On a weak commutativity condition of mappings in fixed point considerations, Publ. Inst. Math. (Beorad) (N S) 32 (46) (1982), 149-153

[12] SINGH, S.L., Coincidence theorems, fixed point theorems and convergence of the sequences of coincidence values, Punjab Univ. J. Math. 19 (1986), 83-97

[13] SINGH, S L and PANT, B D , Coincidence and fixed point theorems for a family of mappings on Menger spaces and extension to uniform spaces, Math. Japon. 33 (1988), 957-973

[14] TIVARI, B ML and SINGH, S L, A note on recent generalizations of Jungck contraction principle, J. UPGC Acad. Soc. 3 (1986), 13-18

[15] PARK, S and BAE, J S, Extensions of a fixed point theorem of Meir and Keeler, Arklv Mat. 19 (1981), 223-228 


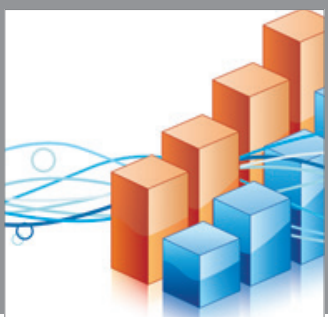

Advances in

Operations Research

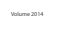

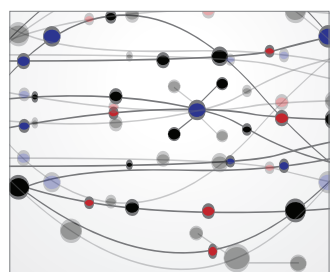

\section{The Scientific} World Journal
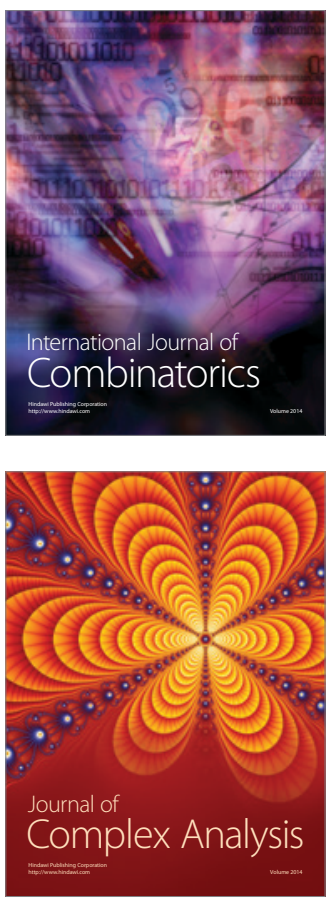

International Journal of

Mathematics and

Mathematical

Sciences
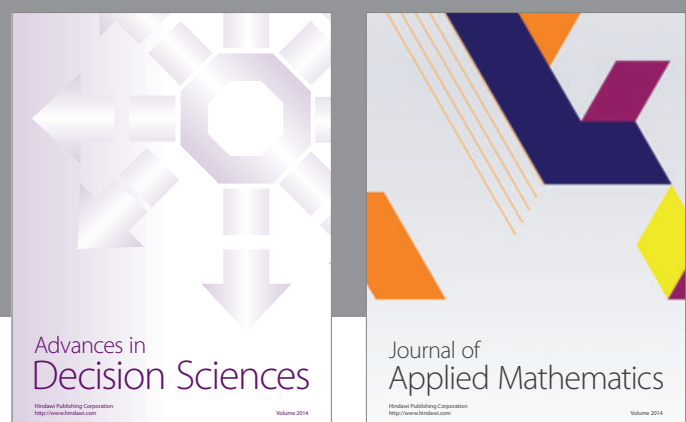

Journal of

Applied Mathematics
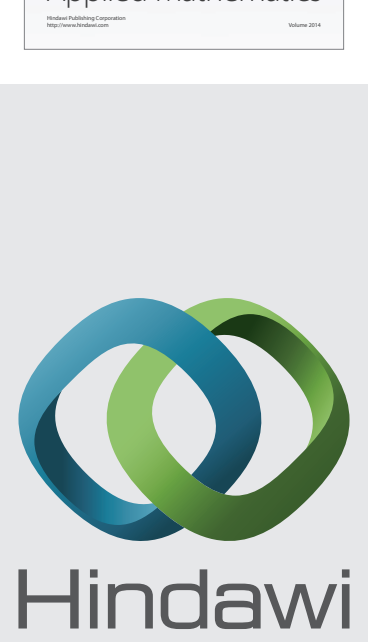

Submit your manuscripts at http://www.hindawi.com
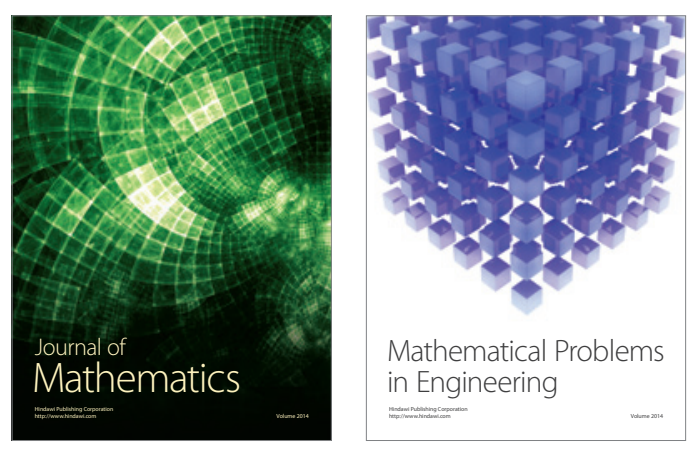

Mathematical Problems in Engineering
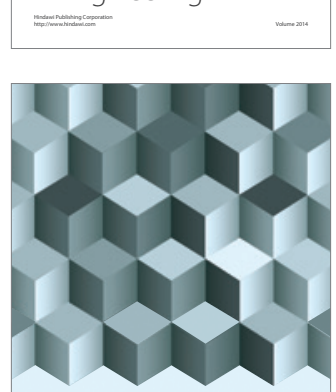

Journal of

Function Spaces
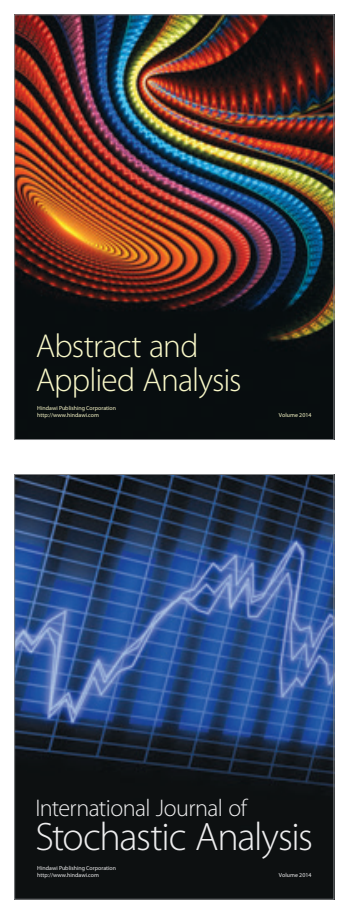

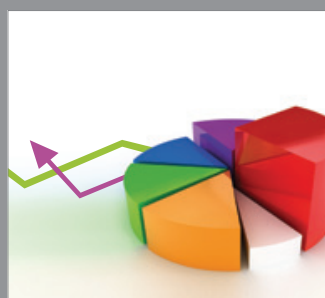

ournal of

Probability and Statistics

Promensencen
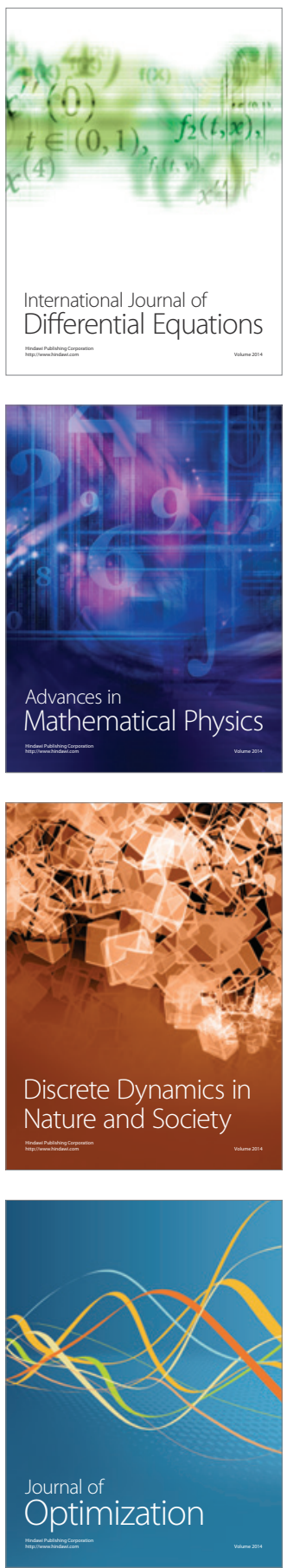